\title{
Nonreciprocal response theory of non-Hermitian mechanical metamaterials: Response phase transition from the skin effect of zero modes
}

\author{
Henning Schomerus $\odot$ \\ Department of Physics, Lancaster University, Lancaster LA1 4YB, United Kingdom
}

(Received 17 August 2019; published 17 January 2020)

\begin{abstract}
Nonreciprocal non-Hermitian systems provide an unconventional localization mechanism of topological zero modes via the non-Hermitian skin effect. While fundamental theoretical characterizations of this effect involve the biorthogonal system of right and left eigenmodes, the recent demonstration of this effect for a zero mode in a robotic metamaterial (Ghatak et al., arXiv:1907.11619) is based on the direct experimental observation of the conventional right eigenvectors. Here I show that such nonreciprocal mechanical metamaterials reveal their underlying biorthogonality in the directly observable response of the system to external excitation. Applied to the experiment, this nonreciprocal response theory predicts that the zero-mode skin effect coincides with an extended phase where the system is highly sensitive to physical perturbations, leading to a diverging response in the limit of a large system.
\end{abstract}

DOI: 10.1103/PhysRevResearch.2.013058

\section{INTRODUCTION}

Nonreciprocal non-Hermitian mechanical metamaterials are a recent innovation $[1,2]$ that enable the experimental study of phenomena arising from the interplay of two principal notions of broken time-reversal symmetry in conservative and dissipative systems. Nonreciprocal media break time-reversal symmetry via effective vector potentials, which translate into striking phenomena such as quantum-Hall-like effects and optical isolation. These effects are absent in the dissipative breaking of time-reversal symmetry by scalar gain and loss, resulting in non-Hermitian physics where resonances acquire finite lifetimes. Nonreciprocity and non-Hermiticity are combined when one considers systems with directed gain or loss mechanisms, allowing them to sustain a finite net flux imbalance, as originally introduced by Hatano and Nelson [3]. An only recently recognized striking consequence is the so-called non-Hermitian skin effect, describing the possibility to localize states at the edge of the system when the imbalance is large enough, which requires one to revisit the well-established bulk boundary correspondence known from Hermitian systems [4-11].

In a recent ground-breaking experiment, an analogous relocalization of a topological zero mode from one edge to the other has been realized in a robotic metamaterial [2]. The experimental observation of this zero-mode skin effect naturally maps out the spatial response of the system, which is linked to the right eigenmodes of the system. On the other hand, the theoretical understanding of the non-Hermitian skin effect [6] highlights the interplay of right and left eigenmodes,

Published by the American Physical Society under the terms of the Creative Commons Attribution 4.0 International license. Further distribution of this work must maintain attribution to the author(s) and the published article's title, journal citation, and DOI. which is much more intricate than in reciprocal systems where both types of eigenmodes are simply related by time-reversal symmetry. In the nonreciprocal case, theory has to invoke biorthogonality relations that involve the complete set of eigenmodes of the system. This leaves the natural question of physical signatures of this intricate interplay.

As I point out in this paper, the left eigenmodes as well as the complete biorthogonal interplay both leave clear signatures that can be directly observed in experiments. These signatures are revealed when one develops the response theory for nonreciprocal media subjected to physical external excitation, which I here exemplify for a general class of systems compassing the robotic metamaterials. The left eigenmodes characterize the strength of the response with respect to the location of the perturbation, while the right eigenmodes characterize the spatial distribution of the response itself, which has been in the focus of the experiments. Intriguingly, the non-Hermitian skin effect of the zero mode then becomes linked with a phase transition, where the sensitivity of the system to low-frequency excitations diverges in the limit of a large system. This extreme sensitivity, which occurs across the whole skin-effect phase and therefore is independent of any spectral singularities, is described by the formal analog of the Petermann factor from quantum-limited noise theory [12-15] and applies generally to a wide class of nonreciprocal non-Hermitian media. Thereby, the mathematical topological phase transition of the non-Hermitian skin effect becomes tied to a physical phase transition in the observable response of the system.

\section{BACKGROUND}

\section{A. Nonreciprocal metamaterials}

I develop the nonreciprocal response theory guided by the robotic metamaterial in Ref. [2], in which $N$ coupled oscillators $\frac{d^{2} \mathbf{x}}{d t^{2}}+M \mathbf{x}=0$ are equipped with a feedback force 
(a)

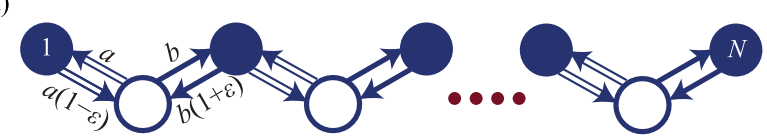

(b)
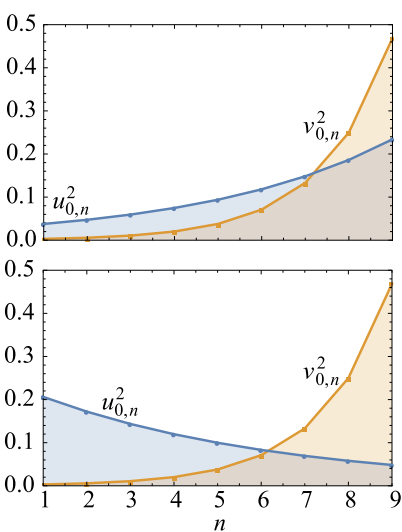

(c)

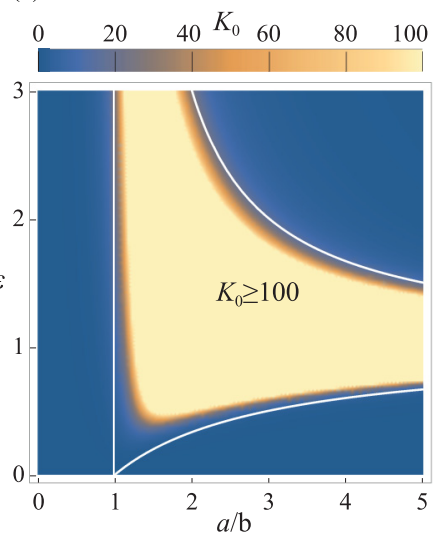

FIG. 1. (a) Nonreciprocal coupling configuration in a nonHermitian robotic metamaterial, using the unfolding Eq. (1) which maps the system onto a nonreciprocal Su-Schrieffer-Heeger chain with a topological zero mode. The full circles represent position degrees of freedom for rotors equipped with feedback, while the open circles relate to the resulting torque, which is not directly observed. This paper develops the nonreciprocal response theory for the rotor dynamics, which enters a phase of extreme sensitivity when the zero mode undergoes a relocalization from one edge to the other in analogy to the non-Hermitian skin effect of bulk states, as shown in (b) for $a=1, b=0.73, N=9$, and $\varepsilon=0.1$ (top), $\varepsilon=0.2$ (bottom). (c) Resulting sensitivity phase diagram for finite $N=9$ in terms of the enhancement factor $K_{0}$, Eq. (14). The white lines enclose the zero-mode skin-effect region.

so that the dynamical matrix $M$ is asymmetric, $M \neq M^{T}$. This realizes the directed couplings of a nonreciprocal nonHermitian system. In the Hermitian limit, the experimental system represents the Kane-Lubensky model of topological mechanics [16-18], which heralds a topological zero mode due to the factorization of $M=Q Q^{T}$ with an $N \times(N-1)$ dimensional matrix $Q$, specifically chosen to have elements $Q_{n m}=-a \delta_{n m}+b \delta_{n-1, m}$ [19]. The robotic metamaterial retains a modified factorization of the form $M=Q R$, where the $(N-1) \times N$ matrix $R$ with elements $R_{n m}=-a(1-$ $\varepsilon) \delta_{n m}+b(1+\varepsilon) \delta_{n, m-1}$ differs from $Q^{T}$. The parameter $\varepsilon$ quantifies the nonreciprocal couplings and induces strong non-Hermitian effects due to the two high-order exceptional points (EPs) at $|\varepsilon|=1 \equiv \varepsilon_{\mathrm{EP}}$, where all the bulk eigenmodes collapse onto a single eigenvector. However, no bulk modes undergo EPs at zero energy and hence do not interfere with the zero mode, as long as $N$ is odd, which we therefore assume throughout. Unfolding the system as

$$
H=\left(\begin{array}{ll}
0 & Q \\
R & 0
\end{array}\right), \quad H^{2}=\operatorname{diag}(Q R, R Q),
$$

the system maps onto the prototypical non-Hermitian variant of a Su-Schrieffer-Heeger chain with nonreciprocal hoppings [5,6,20], illustrated in Fig. 1(a).

\section{B. Dynamical zero mode}

The dynamical modes of the system follow from the eigenvalue equations

$$
M \mathbf{u}_{n}=\Omega_{n} \mathbf{u}_{n}, \quad \mathbf{v}_{n} M=\Omega_{n} \mathbf{v}_{n},
$$

with right and left eigenvectors $\mathbf{u}_{n}$ and $\mathbf{v}_{n}$. Because of the ranks of matrices $Q$ and $R$, there is always a zero mode with $\Omega_{0}=0$, hence $R \mathbf{u}_{0}=0, \mathbf{v}_{0} Q=0$, given by

$$
u_{0, n}=c_{R}\left(\frac{a(1-\varepsilon)}{b(1-\varepsilon)}\right)^{n}, \quad v_{0, n}=c_{L}\left(\frac{a}{b}\right)^{n},
$$

with normalization constants $c_{R}$ and $c_{L}$. The right eigenvector of the zero mode switches its localization position from one edge to the other at $\varepsilon_{1}=\frac{a-b}{a+b}$ and $\varepsilon_{2}=\frac{a+b}{a-b}=\varepsilon_{1}^{-1}$, while the left eigenvector of the zero mode always remains fixed in this design [Fig. 1(b)]. As we will see, the left mode can nonetheless directly be probed via the response of the system. In particular, the sensitivity of the system [Fig. 1(c)] depends critically on the full biorthogonal interplay between the right and left modes, so the invariability of the left mode is highly deceptive.

The main premise of this paper is the expectation that the response of such nonreciprocal non-Hermitian systems to external perturbations should be governed by a properly regularized Green's function:

$$
\hat{G}=\left(\omega^{2} \mathbb{1}-M\right)^{-1} .
$$

Using the spectral decomposition $M=U \hat{\Omega}^{2} U^{-1}$, the Green's function includes the complete spectral information with eigenvalues $\Omega_{n}$ in the diagonal matrix $\hat{\Omega}$, as well as the full biorthogonal structure of eigenmodes with the corresponding right eigenvectors $\mathbf{u}_{n}$ as the columns of $U$ and the left eigenvectors $\mathbf{v}_{n}$ as the rows of $U^{-1}$. In the presence of a zero mode, the Green's function has a double pole around $\omega=0$, complementing the simple poles at the bulk resonance frequencies-but this will not be the origin of the enhanced sensitivity, which instead arises from the emphasized role of mode biorthogonality in the zero-mode skin-effect phase.

\section{RESULTS}

\section{A. Response theory}

To determine the exact role of the Green's function (4), let us develop the detailed response theory of nonreciprocal non-Hermitian mechanical media, where for generality we do not invoke the factorization of $M$ nor assume the existence of a zero mode, hence we also do not restrict aspects such as dimensionality, order, coordination, or range of the couplings. This also anticipates modified designs of nonreciprocal mechanical media that either change the factorization so that left eigenvectors also change their localization position or prevent factorization and remove the zero mode. Furthermore, for generality, we also allow modes to be complex. Focusing on these general features of the system response then reveals the practical role of the right and left eigenvectors and leads to a characterization of the system in terms of its overall sensitivity.

Subject to quasiharmonic external driving force with a fixed force configuration $\mathbf{y}$, the response of the system is 
dictated by

$$
\frac{d^{2}}{d t^{2}} \mathbf{x}+\gamma \frac{d}{d t} \mathbf{x}+M \mathbf{x}+\mathbf{y} \cos (\omega t)=0
$$

where we include a velocity-dependent damping term of strength $\gamma$. Considering some arbitrary initial conditions in the distant past, all finite-frequency components of the initial conditions will be damped out in the quasistationary response, but not those of the zero mode as the damping is velocity dependent. We can remove this residual memory by also considering a finite width of the driving frequency, corresponding to a small imaginary part $\omega \pm i \eta$ in the advanced and retarded sectors of the description.

Using the spectral decomposition $M=U \hat{\Omega}^{2} U^{-1}$ mentioned above, the quasistationary response is then given by

$$
\mathbf{x}(t)=\operatorname{Re}\left[U\left(\frac{e^{-i \hat{\omega} t}}{\hat{\omega}^{2}+i \hat{\omega} \gamma-\hat{\Omega}^{2}}\right) U^{-1}\right] \mathbf{y},
$$

where we set $\hat{\omega}=\omega \mathbb{1}$. This corresponds to the time-frequency Green's function,

$$
G_{n m}(\omega ; t)=\operatorname{Re} \sum_{k} U_{n k}\left(\frac{e^{-i \omega t}}{\omega^{2}+i \omega \gamma-\Omega_{k}^{2}}\right) U_{k m}^{-1},
$$

giving the response at position $n$ for a unit-amplitude excitation at position $m$. This expression naturally captures the spectrum as well as the right and left eigenvectors, where, in particular, the left eigenvectors describe how the response varies as one changes the location of the external drive.

\section{B. Nonreciprocal power spectra}

These features of the system response can be further quantified using the spatially resolved power spectrum for a unit-power excitation positioned at $m$ and detected at $n$,

$$
\begin{aligned}
P_{n m}(\omega) & =2 \lim _{T \rightarrow \infty} \frac{1}{T} \int_{0}^{T} G_{n m}^{2}(\omega ; t) d t \\
& =\sum_{k l} \frac{U_{n k} U_{k m}^{-1}}{\omega^{2}+i \omega \gamma-\Omega_{k}^{2}} \frac{\left(U_{n l} U_{l m}^{-1}\right)^{*}}{\omega^{2}-i \omega \gamma-\Omega_{l}^{* 2}},
\end{aligned}
$$

where we can neglect the interference of the formally counterrotating terms at $\omega=0$ as the power of the excitation has to be renormalized at this point as well (a unit-power excitation has amplitude $\propto \sqrt{2} \cos \omega t$ unless $\omega=0$, where the corresponding amplitude is unity). We also define $P_{m}^{\text {in }}(\omega)=\sum_{n} P_{n m}(\omega)$ for the collective system response to driving at position $m$, and the complementary output spectrum $P_{n}^{\text {out }}(\omega)=\sum_{m} P_{n m}(\omega)$ at position $n$ for driving across the whole system (note that both spectra relate to the observed output power). Close to resonance, where $\omega \approx \Omega_{\bar{k}}$ for a specific overlapping mode $\bar{k}$ [21],

$$
P_{m}^{\mathrm{in}}(\omega) \approx \frac{\left(U^{\dagger} U\right)_{\bar{k} \bar{k}}}{\left(\omega^{2}-\Omega_{\bar{k}}^{2}\right)^{2}+\omega^{2} \gamma^{2}}\left|U_{\bar{k} m}^{-1}\right|^{2}
$$
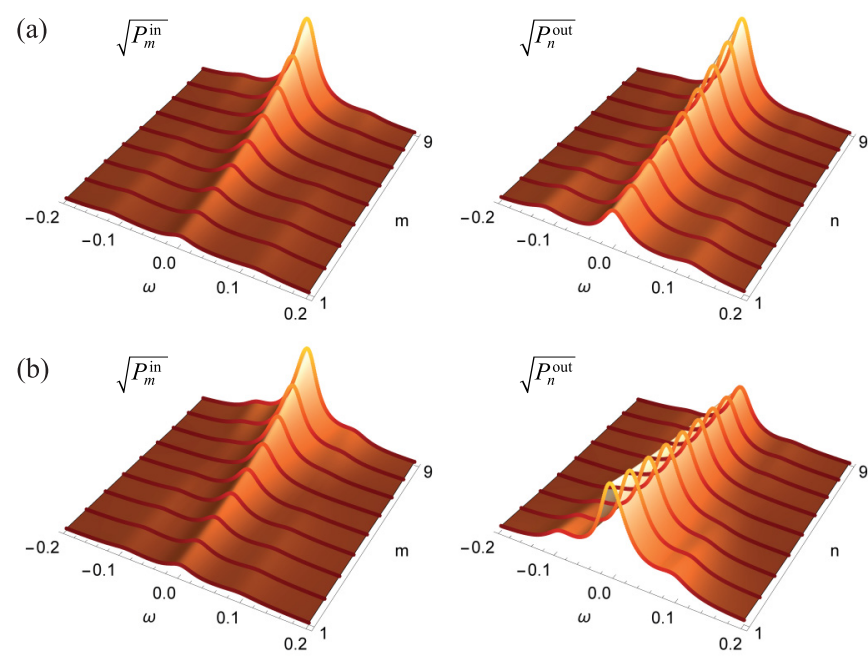

FIG. 2. (a) Power amplitudes $\sqrt{P_{m}^{\text {in }}(\omega)}$ (left) and $\sqrt{P_{n}^{\text {out }}(\omega)}$ (right) [arb. units] for the nonreciprocal non-Hermitian robotic metamaterial with $N=9$ components and parameters $a=1, b=0.73$, $\varepsilon=0.1$, in the frequency range $|\omega| \leqslant 0.2$. The guiding surfaces are interpolated in the discrete indices $n$ or $m$. The central ridge corresponds to the zero mode and maps out the corresponding left and right eigenvectors, respectively. The secondary ridges arise from the principal bulk mode, which resides at $\Omega_{1}=0.121$. (b) Same for $\varepsilon=0.2$, beyond the critical value $\varepsilon_{1}=0.156$ at which the right eigenvector of the zero mode switches its localization center. The neighboring bulk mode resides at $\Omega_{1}=0.095$. The results are based on the regularization Eq. (12) with $\eta=0.025$.

is then proportional to the intensity profile of the left eigenvector, while

$$
P_{n}^{\text {out }}(\omega) \approx \frac{\left(U^{-1} U^{-\dagger}\right)_{\bar{k} \bar{k}}}{\left(\omega^{2}-\Omega_{\bar{k}}^{2}\right)^{2}+\omega^{2} \gamma^{2}}\left|U_{n \bar{k}}\right|^{2}
$$

is proportional to the intensity profile of the right eigenvector.

Note that, formally, the regularized results with a finite damping rate $\gamma$ are similar to the aforementioned implied frequency shift $\omega \rightarrow \omega \pm i \eta$ in the advanced and retarded Green's functions, which translates to the corresponding sectors of the power spectrum as

$$
P_{n m}(\omega)=\sum_{k l} \frac{U_{n k} U_{k m}^{-1}\left(U_{n l} U_{l m}^{-1}\right)^{*}}{\left((\omega-i \eta)^{2}-\Omega_{k}^{2}\right)\left((\omega+i \eta)^{2}-\Omega_{l}^{2}\right)} .
$$

However, the regularizations differ around $\omega=0$, where the physical velocity-dependent damping is ineffective. In contrast, the regularization Eq. (12) corresponds to a finite width in the frequency of the driving force itself. In Fig. 2, we illustrate the response of representative system configurations in terms of this frequency regularization. Using the values $a=1$ and $b=0.73$ from the experiment [2], the zero-mode skin effect occurs at $\varepsilon_{1}=0.156$. The figure clearly shows how the system response is enhanced at opposite edges for values on either side of this transition, following the relocalization of the right eigenmode. In contrast, the sensitivity of the system follows the invariable profile of the left eigenmode. 


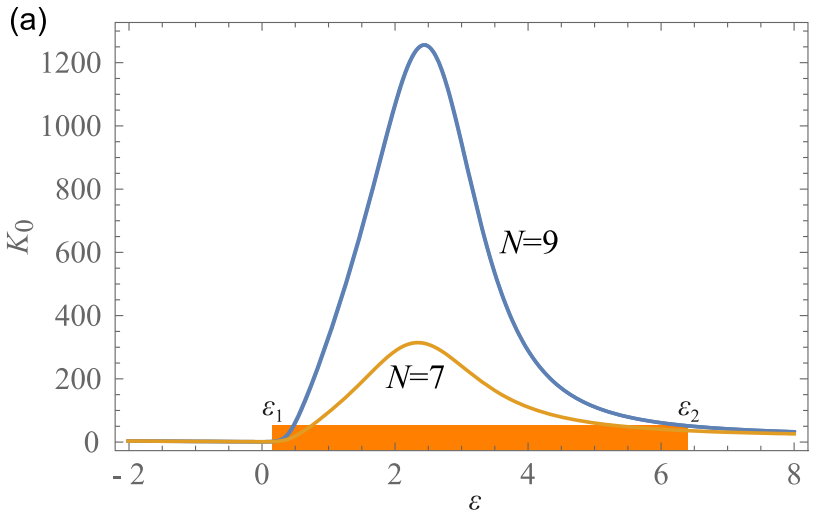

(b)
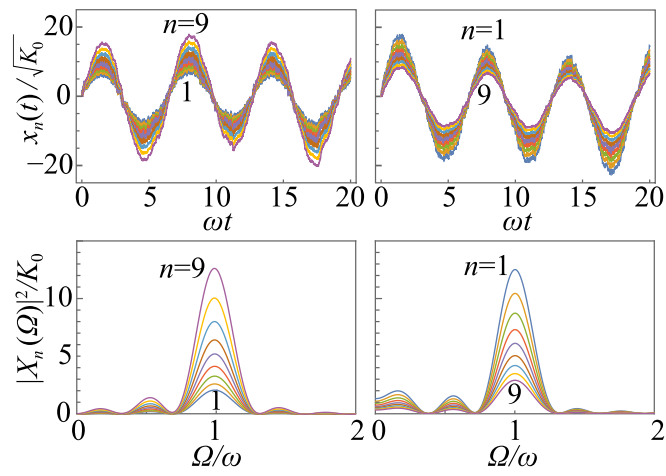

FIG. 3. (a) Petermann factor $K_{0}$ describing the enhanced sensitivity of the zero mode as a function of the non-Hermiticity parameter $\varepsilon$, for finite systems with $N=7,9$ and $a=1$ and $b=0.73$. The skin-effect phase of the zero mode is shaded on the horizontal axis. (b), (c) Simulated time traces $x_{n}(t)$ (top) and corresponding empirical power spectrum obtained from $X_{n}(\Omega)=(2 \pi T)^{-1} \int_{0}^{T} d t x_{n}(t) e^{-i \Omega t}$ (bottom) from a force $F_{5}=\cos \omega t+\xi(t)$ applied to the central rotor of the system of length $N=9$, at slightly off-resonant driving frequency $\omega=0.01$, stochastic noise $\xi(t)$ of variance $\eta=0.025$, and damping $\gamma=0.5$. In (b), $\varepsilon=0.1$ while in (c) $\varepsilon=0.2$. Both the quasistationary response as well as the empirical power spectrum scale with the Petermann factor.

\section{Enhanced sensitivity}

We now turn to the characterization of the system in terms of its overall sensitivity, which is captured by the total power spectrum $P^{\text {tot }}(\omega)=\sum_{n m} P_{n m}(\omega)$. Notably, near resonance, the overall response

$$
P^{\mathrm{tot}}(\omega) \approx \frac{K_{\bar{k}}}{\left(\omega^{2}-\Omega_{\bar{k}}^{2}\right)^{2}+\omega^{2} \gamma^{2}}
$$

is then weighted by a factor

$$
K_{\bar{k}}=\left(U^{\dagger} U\right)_{\bar{k} \bar{k}}\left(U^{-1} U^{-\dagger}\right)_{\bar{k} \bar{k}} .
$$

Mathematically, $K_{\bar{k}} \geqslant 1$ represents a condition number quantifying the mode nonorthogonality [22], while physically it signifies the ensuing enhanced sensitivity of the system to perturbations, in analogy to the Petermann factor from quantumlimited noise theory [12-14], which quantifies excess noise. The Petermann factor has been studied extensively for reciprocal non-Hermitian systems [15,23-25], where $U^{-1}=U^{T}$ so it can be calculated using only the right eigenvectors. In the present nonreciprocal case, however, we encounter a situation where this enhanced sensitivity involves the complete biorthogonal interplay of right and left eigenvectors.

Applying these results to the experimental setting, we first observe that the zero mode can have a strikingly large Petermann factor, despite its symmetry protection that distinguishes it from all other modes. The known mode profiles Eqs. (3) deliver the expression

$$
K_{0}=\frac{\left[\sum_{n=1}^{N}(a / b)^{2 n}\right]\left[\sum_{n=1}^{N}(a(1-\varepsilon) / b(1+\varepsilon))^{2 n}\right]}{\left[\sum_{n=1}^{N}\left(a^{2}(1-\varepsilon) / b^{2}(1+\varepsilon)\right)^{n}\right]^{2}},
$$

which is large in the zero-mode skin-effect phase $\varepsilon_{1}<\varepsilon<\varepsilon_{2}$. The situation for finite $N=7,9$ is illustrated in Fig. 3(a), which verifies that the Peterman factor rapidly increases when entering the phase with $\varepsilon>\varepsilon_{1}$, and again drops down leaving the phase at $\varepsilon>\varepsilon_{2}$. Equipped with the skin effect, the zero mode can therefore react strongly to physical excitations, increasing its visibility in the experiments. This is further illustrated in Figs. 3(b) and 3(c), which show that the scaling of the sensitivity with $K_{0}$ can be determined even under slightly off-resonant, noisy driving with damping, from a single finite trace of the dynamics - both from the amplitude of the response as well as from the empirical power spectrum.

\section{Response phase transition}

Notably, in the limit of a large system $N \rightarrow \infty$, the Petermann factor remains finite only outside the skin-effect phase, where

$$
K_{0}^{N \rightarrow \infty}=\frac{\left[a^{2}(1-\varepsilon)-b^{2}(1+\varepsilon)\right]^{2}}{\left[a^{2}-b^{2}\right]\left[a^{2}(1-\varepsilon)^{2}-b^{2}(1+\varepsilon)^{2}\right]},
$$

while $K_{0}$ diverges exponentially with increasing system size in the range $\varepsilon_{1}<\varepsilon<\varepsilon_{2}$. This fully reveals the advertised transition to a phase of highly sensitive response, which is intimately tied to the non-Hermitian skin effect of the zero mode. This correspondence emerges quickly across the whole parameter space already for moderate system sizes, as illustrated in Fig. 1(c).

Note that in common non-Hermitian settings, diverging sensitivities can occur via EPs [26], i.e., spectral singularities at fine-tuned parameters in which eigenmodes collapse and the system becomes defective. The sensitive response phase identified here, however, is not tied to this mechanism. In particular, whilst being very large for a finite system across the whole skin-effect phase, the sensitivity of the zero mode does not peak at the highorder bulk EP $\varepsilon_{\mathrm{EP}}=1$, which strongly affects all the other modes according to $\left.K_{n \neq 0} \sim\left[\left(\varepsilon_{\mathrm{EP}}-\varepsilon\right) / 2\right)\right]^{1-N} / N^{2}$ for $\varepsilon \rightarrow$ $\varepsilon_{\mathrm{EP}}[27]$. Furthermore, the transition occurs while the complete resonance spectrum remains real, hence does not resemble, e.g., the spontaneous breaking of non-Hermitian symmetries as observed in parity-time (PT)-symmetric systems [28]. 


\section{CONCLUSIONS}

In summary, probing the dynamical response of nonHermitian nonreciprocal metamaterials gives directly observable insights into the right and left eigenmodes, while the overall sensitivity of the system is governed by the full biorthogonal interplay between both sets of modes. As we demonstrated for the example of a recently realized robotic metamaterial, the non-Hermitian skin effect changing the localization position of a zero mode directly correlates with a phase transition in which the medium becomes critically sensitive to perturbations. These observations may be useful for sensing applications, which in contrast to earlier proposals invoking non-Hermiticity [26,29] would not rely on the closeness to an EP and hence do not require the fine-tuning of parameters.

The general expressions of nonreciprocal non-Hermitian response theory apply to linear systems with arbitrary dynamical matrix $M$, which can model linear systems of different dimensionality, coordination number, or range and disorder in the couplings, and also serve as the starting point to derive continuum descriptions from microscopic models. Given the typical structures encountered for the right and left zero modes in the prototypical models studied so far, we expect this phase transition to be a general feature of nonreciprocal systems exhibiting a corresponding non-Hermitian skin effect, and possibly also extend to the skin effect of nonzero modes, as well as to recent nonreciprocal and reciprocal variants of non-Hermitian topoelectric circuits [30,31]. From a more fundamental perspective, the extreme sensitivity in the non-Hermitian skin-effect phase outlines a practical limitation to stabilize non-Hermitian phases, in analogy to what has transpired, e.g., for PT-symmetric systems based on their sensitivity to quantum fluctuations $[32,33]$.

\section{ACKNOWLEDGMENTS}

The author acknowledges funding by EPSRC via Grant No. EP/P010180/1 and Programme Grant No. $\mathrm{EP} / \mathrm{N} 031776 / 1$, as well as the hospitality of NORDITA where this work was carried out.
[1] M. Brandenbourger, X. Locsin, E. Lerner, and C. Coulais, Non-reciprocal robotic metamaterials, Nat. Commun. 10, 4608 (2019).

[2] A. Ghatak, M. Brandenbourger, J. van Wezel, and C. Coulais, Observation of non-Hermitian topology and its bulk-edge correspondence, arXiv:1907.11619.

[3] N. Hatano and D. R. Nelson, Localization Transitions in Non-Hermitian Quantum Mechanics, Phys. Rev. Lett. 77, 570 (1996).

[4] Y. Xiong, Why does bulk boundary correspondence fail in some non-Hermitian topological models, J. Phys. Commun. 2, 035043 (2018).

[5] S. Yao and Z. Wang, Edge States and Topological Invariants of Non-Hermitian Systems, Phys. Rev. Lett. 121, 086803 (2018).

[6] F. K. Kunst, E. Edvardsson, J. C. Budich, and E. J. Bergholtz, Biorthogonal Bulk-Boundary Correspondence in Non-Hermitian Systems, Phys. Rev. Lett. 121, 026808 (2018).

[7] S. Lieu, Topological phases in the non-Hermitian Su-SchriefferHeeger model, Phys. Rev. B 97, 045106 (2018).

[8] A. McDonald, T. Pereg-Barnea, and A. A. Clerk, PhaseDependent Chiral Transport and Effective Non-Hermitian Dynamics in a Bosonic Kitaev-Majorana Chain, Phys. Rev. X 8, 041031 (2018).

[9] V. M. Martinez Alvarez, J. E. Barrios Vargas, and L. E. F. Foa Torres, Non-Hermitian robust edge states in one dimension: Anomalous localization and eigenspace condensation at exceptional points, Phys. Rev. B 97, 121401(R) (2018).

[10] C. H. Lee and R. Thomale, Anatomy of skin modes and topology in non-Hermitian systems, Phys. Rev. B 99, 201103(R) (2019).

[11] W. Brzezicki and T. Hyart, Hidden Chern number in onedimensional non-Hermitian chiral-symmetric systems, Phys. Rev. B 100, 161105 (2019).

[12] A. E. Siegman, Excess spontaneous emission in non-Hermitian optical systems. I. Laser amplifiers, Phys. Rev. A 39, 1253 (1989).
[13] M. Patra, H. Schomerus, and C. W. J. Beenakker, Quantumlimited linewidth of a chaotic laser cavity, Phys. Rev. A 61, 023810 (2000).

[14] M. V. Berry, Mode degeneracies and the Petermann excessnoise factor for unstable lasers, J. Mod. Opt. 50, 63 (2003).

[15] A. Pick, B. Zhen, O. D. Miller, C. W. Hsu, F. Hernandez, A. W. Rodriguez, M. Soljačić, and S. G. Johnson, General theory of spontaneous emission near exceptional points, Opt. Express 25, 12325 (2017)

[16] C. L. Kane and T. C. Lubensky, Topological boundary modes in isostatic lattices, Nat. Phys. 10, 39 (2014).

[17] B. G. Chen, N. Upadhyaya, and V. Vitelli, Nonlinear conduction via solitons in a topological mechanical insulator, PNAS 111, 13004 (2014).

[18] S. D. Huber, Topological mechanics, Nat. Phys. 12, 621 (2016).

[19] Studying the eigenmodes of the matrix $Q$ itself allows one to identify modes of self-stress, which differ from the right and left eigenmodes of the dynamical matrix $M$ [16].

[20] In principle, such models can also be generated in driven reciprocal systems - see, for example, S. Longhi, D. Gatti, and G. Della Valle, Robust light transport in non-Hermitian photonic lattices, Sci. Rep. 5, 13376 (2015).

[21] In the following expressions, we assume for transparency real bare resonance frequencies, as these give rise to the most visible response features and naturally encompass the zero mode. From here the generalization to complex frequencies is straightforward and only results in a further broadening, but retains the role of the right and left eigenvectors as well as the Petermann factor Eq. (14).

[22] J. T. Chalker and B. Mehlig, Eigenvector Statistics in NonHermitian Random Matrix Ensembles, Phys. Rev. Lett. 81, 3367 (1998).

[23] H. Schomerus, K. Frahm, M. Patra, and C. Beenakker, Quantum limit of the laser line width in chaotic cavities and statistics of residues of scattering matrix poles, Physica A 278, 469 (2000). 
[24] Y. V. Fyodorov and B. Mehlig, Statistics of resonances and nonorthogonal eigenfunctions in a model for single-channel chaotic scattering, Phys. Rev. E 66, 045202(R) (2002).

[25] G. Yoo, H.-S. Sim, and H. Schomerus, Quantum noise and mode nonorthogonality in non-Hermitian PT-symmetric optical resonators, Phys. Rev. A 84, 063833 (2011).

[26] J. Wiersig, Enhancing the Sensitivity of Frequency and Energy Splitting Detection by Using Exceptional Points: Application to Microcavity Sensors for Single-Particle Detection, Phys. Rev. Lett. 112, 203901 (2014).

[27] With periodic boundary conditions, the bulk spectrum also displays exceptional points at $\varepsilon_{1}$ and $\varepsilon_{2}$, but these are not affecting the system with open boundary conditions [6].

[28] R. El-Ganainy, K. G. Makris, M. Khajavikhan, Z. H. Musslimani, S. Rotter, and D. N. Christodoulides, NonHermitian physics and PT symmetry, Nat. Phys. 14, 11 (2018).
[29] W. Chen, Ş. Kaya Özdemir, G. Zhao, J. Wiersig, and L. Yang, Exceptional points enhance sensing in an optical microcavity, Nature 548, 192 (2017).

[30] T. Helbig, T. Hofmann, S. Imhof, M. Abdelghany, T. Kiessling, L. W. Molenkamp, C. H. Lee, A. Szameit, M. Greiter, and R. Thomale, Observation of bulk boundary correspondence breakdown in topolectrical circuits, arXiv:1907.11562.

[31] T. Hofmann, T. Helbig, F. Schindler, N. Salgo, M. Brzezińska, M. Greiter, T. Kiessling, D. Wolf, A. Vollhardt, A. Kabaši, C. H. Lee, A. Bilušić, R. Thomale, and T. Neupert, Reciprocal skin effect and its realization in a topolectrical circuit, arXiv:1908.02759.

[32] H. Schomerus, Quantum Noise and Self-Sustained Radiation of PT-Symmetric Systems, Phys. Rev. Lett. 104, 233601 (2010).

[33] S. Scheel and A. Szameit, PT-symmetric photonic quantum systems with gain and loss do not exist, EPL 122, 34001 (2018). 